\title{
Untangling the spin of a dark boson in $Z$ decays
}

\author{
Andrea Comelato $\circledast^{1}$ and Emidio Gabrielli $\circledast^{1,2,3}$ \\ ${ }^{1}$ Department of Physics-Theoretical Section, University of Trieste, \\ Strada Costiera 11, 34151 Trieste, Italy \\ ${ }^{2}$ INFN, Sezione di Trieste, Via Valerio 2, 34127 Trieste, Italy \\ ${ }^{3}$ NICPB, Rävala 10, Tallinn 10143, Estonia
}

(Received 7 June 2020; accepted 22 July 2020; published 30 July 2020)

\begin{abstract}
We analyze the $Z$-boson decay $Z \rightarrow \gamma X$ into a photon $(\gamma)$ plus a hypothetical light boson $(X)$ belonging to a dark or secluded sector. Because of its feeble interactions with Standard Model fields, this dark boson behaves as missing energy in the detector. We consider for $X$ the cases of spin-1 (massless dark-photon), spin-0 (axionlike), and spin-2 (gravitonlike) particles and explore the way to untangle its spin origin. All these scenarios predict a universal signature for this decay, characterized by a single monochromatic photon in the $Z$ center of mass, with energy about half of the $Z$ mass, plus a neutrinolike missing energy associated with the $X$ boson. We show that if the $Z \rightarrow \gamma X$ signal should be discovered at $e^{+} e^{-}$colliders, the angular distribution of the monochromatic photon in $e^{+} e^{-} \rightarrow Z \rightarrow \gamma X$ can provide a clean probe to discriminate between the $J=1$ and alternative $J=0 / 2$ spin nature of the dark boson.
\end{abstract}

DOI: $10.1103 /$ PhysRevD.102.015028

\section{INTRODUCTION}

The lack of any experimental evidence at the LHC for a heavy new physics (NP) above the TeV scale [1], as expected for the many NP scenarios in beyond the Standard Model (SM) theory, is changing our perspective on the search for a NP. The accessible sector of NP could instead be made up of light new particles, feebly coupled to SM fields, as predicted by scenarios with dark or secluded sectors beyond the SM, where, for instance, the candidate(s) for dark matter might reside. The dark sector, consisting of new particles which are singlets under the SM gauge interactions, can indeed have its own long-range interactions, characterized by massless or very light mediators like the dark photon, the quantum field associated with a $U(1)_{D}$ gauge invariance in the dark sector. These scenarios have motivated the search for weakly coupled light particles, as can be seen in the many theoretical and experimental works on this subject [2].

In this framework, we focus on the effective couplings of a light and long-lived neutral $X$ boson with the neutral sector of electroweak gauge bosons of the SM. In particular, we explore, in a model-independent way, the production of $X$ by means of the $Z$-boson decay into

Published by the American Physical Society under the terms of the Creative Commons Attribution 4.0 International license. Further distribution of this work must maintain attribution to the author(s) and the published article's title, journal citation, and DOI. Funded by SCOAP ${ }^{3}$.

$$
Z \rightarrow \gamma X
$$

where $X$ it is assumed to behave as missing energy in the detector.

The striking experimental signature of this decay, in the $Z$ rest frame, is then characterized by an isolated monochromatic photon, with energy (almost) half of the $Z$ mass, and missing energy with (almost) vanishing invariant mass for a massless (massive) $X$.

The best place to look for the process in Eq. (1) is at $e^{+} e^{-}$colliders, where the main characteristic of the signature is maintained, although the monochromaticity of the photon is slightly spread by the initial bremsstrahlung radiation. Moreover, rare $Z$ decays are expected to be investigated at the Future Circular Collider (FCC-ee), with its projected production of $10^{13} \mathrm{Z}$ bosons [3]. This process was already explored at the experimental level at the Large Electron-Positron Collider (LEP) via

$$
e^{+} e^{-} \rightarrow Z \rightarrow \gamma+X,
$$

where $X$ stands for no other detected neutral particles. Negative evidence for this signal, set a limit of $10^{-6}$ at the 95\% C.L. on the corresponding branching ratio (BR), in the case of a massless final state $X$ [4]. On the other hand, at hadron colliders this signal would be rather difficult to detect due to the challenging reconstruction of the $Z$-invariant mass and the large background of soft jets faking the missing energy.

This process was recently analyzed in the case of $X$ as a massless dark photon [5]. The dark-photon scenario has 
been extensively analyzed in the literature, mainly for the massive case, and it is also the subject of many current experimental searches; see [6,7] for a more recent review. Most of the experimental searches focus on massive dark photons, where the $U(1)_{D}$ gauge field generates, through a potential kinetic mixing with the photon, a tree-level (millicharged) interaction with ordinary charged SM particles. On the other hand, for a massless dark photon the kinetic mixing can be fully rotated away, leading to darkphoton interactions with ordinary matter mediated by effective higher-dimensional operators [8]. The leading coupling of a massless dark photon to SM charged particles is provided by the magnetic- and electric-dipole interactions [8,9], including the flavor-changing ones [10]. Phenomenological implications of massless dark-photon scenarios were recently explored in the framework of Higgs boson [11] and rare kaon decays [12].

Recently, in [5] it has been shown that $Z$ can decay at one loop into a photon and massless dark photon without violating the Landau-Yang theorem [13] due to the fact that the dark and ordinary photons are distinguishable particles. An upper limit on the viable BR for the decay $Z \rightarrow \gamma \bar{\gamma}$ has been estimated to be of the order of $O\left(10^{-9}\right)$ [5] in the framework of a simplified model of the dark sector. These results also hold for a massive dark photon due to its own magnetic-dipole interactions with SM fields. ${ }^{1}$

We will explore here the possibility that other $X$-spin configurations can mimic the same signature of a massless dark photon in Eq. (1) and show how to disentangle a genuine spin-1 dark-photon signal against possible $X$ candidates with different integer spins. We will assume an uncertainty of the order of a $1 \mathrm{GeV}$ in the invariant mass of the missing energy, mainly due to the detector performance in the reconstruction of the missing mass. Therefore, as an alternative to the massless dark photon, we consider at the phenomenological level hypothetical scenarios of spin- 0 and spin- 2 particles with masses $m_{X}$ below the $1 \mathrm{GeV}$ scale which are inspired by known theoretical frameworks.

In this respect, we consider first, as an alternative to the dark photon, $X$ to be a light axionlike particle (ALP) in both scalar and pseudoscalar scenarios. ALPs have been predicted in several SM extensions, motivated mainly by the solution to the strong- $C P$ problem, where the ALP is a QCD axion [15], or associated with pseudoNambu-Goldstone bosons corresponding to spontaneously broken continuous symmetries (in either the visible or the dark sector), as well as to a moduli field in string models [16-19]. The phenomenological aspects of the

\footnotetext{
${ }^{1}$ The $Z \rightarrow \gamma V$ decay was also explored in [14] for a massive vectorial field $V$ (not exactly a dark photon) coupled with anomalous nonconserved $U(1)$ currents by gauging anomalous $U(1)$ symmetries of the SM [14].
}

ALPs have been extensively investigated in recent years, especially collider search of ALPs [20-22]. The most severe constraints on the ALP couplings are in the range of masses below the $\mathrm{MeV}$ scale, mainly due to low energy observables and constraints from astrophysics and cosmology [21].

The $Z$ decay process in Eq. (1), with $X=a$ as the ALP, has been considered in the literature [23], More recently, a dedicated study on the sensitivity of the $Z \rightarrow a \gamma$ decay at the LHC and future colliders, via visible ALP decays into two photons and/or lepton pairs [20,21], has been explored for various mass ranges. Present constraints on the effective scale, based mainly on previous LEP analyses on ALP visible decays, allows for $\mathrm{BR}(Z \rightarrow a \gamma)$ as large as $O\left(10^{-4}\right)$ for the range of masses $100 \mathrm{MeV} \lesssim m_{a} \lesssim 1 \mathrm{GeV}$. We will show that, under the requirement for the ALP to behave as missing energy in the detector, stronger constraints on the BR for this decay apply that could reach $O\left(10^{-6}\right)$ for masses of the order of $1 \mathrm{GeV}$. This is also consistent with the LEP bound [4] that applies to the corresponding signature. These results can be easily generalized to ALP in both the scalar and pseudoscalar cases. Therefore, a large number of viable events for $Z \rightarrow a \gamma$ at future $e^{+} e^{-}$colliders with high luminosity are expected that could be competitive with the corresponding ones from the $Z \rightarrow \gamma \bar{\gamma}$ signature.

Next, we consider a more exotic scenario for $X$ as an ultralight massive spin-2 particle $G$. Fundamental massive spin-2 fields have been predicted in several extensions of gravity theories, like the massive Kaluza-Klein (KK) excitations of the standard massless graviton in quantum gravity theories in large extra dimensions (ArkaniHamed-Dimopoulos-Dvali (ADD) [24] and RandalSundrum [25] scenarios), as well as the massive graviton in bimetric theories [26-28]. For the purposes of this analysis, we do not make any assumptions about the origin of this field. Since we are interested only in the phenomenological implications of $Z \rightarrow \gamma G$ decay, we restrict the analysis to the effects of the linear theory (with an on-shell $G$ field as an external source) in flat space-time, a characteristic common to many extended gravity scenarios. For consistency, we assume the spin-2 field to be universally coupled to the energy-momentum tensor of the SM fields, as for the linear gravitonlike coupling to the SM fields, with an effective scale $\Lambda_{G}$. Then the effective $Z \gamma G$ vertex is predicted as a function of $\Lambda_{G}$ to be finite, induced at one loop by SM fields running as virtual particles.

To avoid constraints from short-range gravity experiments (see [29] for a recent review) and mimic a neutrinolike signature, we restrict its mass to lie the range $\mathrm{eV} \lesssim m_{G} \lesssim 1 \mathrm{GeV}$, with an effective scale $\Lambda_{G} \geq \mathrm{TeV}$, and require that it does not decay inside the detector. We will show that, for a spin-2 particle subject to these 
specific constraints, predictions for $\operatorname{BR}(Z \rightarrow \gamma G)$ as large as $O\left(10^{-8}\right)$ are possible, and thus in the sensitivity range considered here for $Z \rightarrow \gamma X$. $^{2}$

Now, assuming the process in Eq. (1) will be observed with a BR in the sensitivity range of $\operatorname{BR}(Z \rightarrow \gamma X) \sim$ $10^{-12}-10^{-6}$ given the possibility that $X$ might belong to one of these scenarios, one may wonder if its spin nature could be disentangled by analyzing the angular distributions of the outgoing photon. Clearly, the answer is no if the $Z$ boson is unpolarized. Indeed, in the unpolarized $Z \rightarrow \gamma X$ decay the photon will be isotropically distributed independently of the spin nature of the $X$ particle. However, a nontrivial angular distribution of the photon that depends on the $X$ spin can appear in the case of polarized $Z$ decays. Remarkably, one of the main features of the $e^{+} e^{-}$colliders at the resonant $Z$ peak is that the on-shell $Z$ boson is always produced polarized, thus transmitting the $Z$-spin correlations to the final state. In this regard, we will show that the angular distribution of the monochromatic photon in the $e^{+} e^{-} \rightarrow Z \rightarrow \gamma X$ process at the $Z$ peak can offer a clean probe to untangle the spin-1 nature of the $X$ boson against other possible spin- $0 / 2$ interpretations.

The paper is organized as follows. In Sec. II, we give the expressions for the effective Lagrangians relevant to the decay $Z \rightarrow \gamma X$ for the three spin scenarios mentioned above, providing the corresponding amplitudes and total rates, as well as a discussion on the corresponding allowed range of branching ratios. In Sec. III, we analyze the angular distributions of polarized $Z$ decays in each spin- $X$ scenario, while the corresponding results for a $Z$ produced in a resonant $s$-channel at $e^{+} e^{-}$colliders is presented in Sec. IV. Finally, our conclusions are reported in Sec. V.

\section{EFFECTIVE LAGRANGIANS AND AMPLITUDES}

\section{A. Spin 1: Massless dark photon}

We consider here the case of $X$ as a massless dark photon $\bar{\gamma}$ which is effectively coupled to the photon $\gamma$ and $Z$ gauge boson. A generalization to the massive dark photon in the limit of small mass is straightforward. We first recall the main results obtained in [5].

The lowest-dimensional gauge-invariant Lagrangian ( $C P$ even) for the leading contribution to the effective $Z \gamma \bar{\gamma}$ vertex was derived in [5]. We parametrize this Lagrangian as

\footnotetext{
${ }^{2}$ The decay in Eq. (1) with a massive spin-2 $X$ was analyzed in [30] in the framework of the ADD scenario [24], predicting a viable BR of the order of $O\left(10^{-11}\right)$ for $D=2$. However, there the signature differs from the one analyzed here due to the absence of events with a monochromatic photon characteristic. Indeed, in ADD this decay can be observed only as inclusive production of an (almost) continuum spectrum of KK graviton excitations, behaving as missing energy, thus reflected in an (almost) continuum photon spectrum.
}

$$
\mathcal{L}_{\text {eff }}=\frac{e}{\Lambda M_{Z}} \sum_{i=1}^{3} C_{i} \mathcal{O}_{i}(x)
$$

where $e$ is the unit of electric charge, $\Lambda$ is the scale of the new physics, the dimension- 6 operators $\mathcal{O}_{i}$ are given by

$$
\begin{aligned}
& \mathcal{O}_{1}(x)=Z_{\mu \nu} \tilde{B}^{\mu \alpha} A^{\nu}{ }_{\alpha}, \\
& \mathcal{O}_{2}(x)=Z_{\mu \nu} B^{\mu \alpha} \tilde{A}^{\nu}{ }_{\alpha}, \\
& \mathcal{O}_{3}(x)=\tilde{Z}_{\mu \nu} B^{\mu \alpha} A^{\nu}{ }_{\alpha},
\end{aligned}
$$

the field strengths $F_{\mu \nu} \equiv \partial_{\mu} F_{\nu}-\partial_{\nu} F_{\mu}$, for $F_{\mu \nu}=$ $(Z, B, A)_{\mu \nu}$, correspond to the $Z$-boson $\left(Z_{\mu}\right)$, dark-photon $\left(B_{\mu}\right)$, and photon $\left(A_{\mu}\right)$ fields, respectively, and $\tilde{F}^{\mu \nu} \equiv$ $\varepsilon^{\mu \nu \alpha \beta} F_{\alpha \beta}$ is the dual field strength. The expression for the coefficients $C_{M}$ in Eq. (7), derived in [5], can be found in the Appendix.

As mentioned in the Introduction, the Landau-Yang theorem [13] can be avoided in $Z \rightarrow \gamma \bar{\gamma}$ due the fact that the photon and the massless dark photon are distinguishable particles. Less obvious is how this effective vertex can be generated from a UV theory. In [5] it has been demonstrated that the above Lagrangian in Eq. (3) arises at low energy as an effective one-loop contribution, with SM fermions running in the loop, because the dark photon does not have tree-level couplings with SM fields. Indeed, the leading coupling of a massless dark photon to charged SM fermions is via magnetic- or electric-dipole operators, namely,

$$
\mathcal{L}_{\text {dipole }}=\sum_{f} \frac{e_{D}}{2 \Lambda} \bar{\psi}_{f} \sigma_{\mu \nu}\left(d_{M}^{f}+i \gamma_{5} d_{E}^{f}\right) \psi_{f} B^{\mu \nu},
$$

where $B^{\mu \nu}$ is the corresponding $U(1)_{D}$ field strength of the dark-photon field, the sum runs over all the SM fields, $e_{D}$ is the $U_{D}(1)$ dark elementary charge (we assume universal couplings), $\Lambda$ is the effective scale of the dark sector, and $\psi_{f}$ is a generic SM fermion field. The scale $\Lambda$ appearing in Eq. (3) is the same as that in Eq. (7). The magnetic- and electric-dipole coefficients $d_{M}^{f}$ and $d_{E}^{f}$, respectively, can be computed from a renormalizable UV completion theory for the dark sector [5].

If the dark photon would have been coupled at tree level with SM charged fermions (as with the ordinary photon or the millicharge couplings of a massive dark photon), the loop contribution would have been zero for each fermion running in the loop, which is in agreement with what is predicted by the Landau-Yang theorem. Therefore, from the point of view of a renormalizable UV completion of the theory, the effective Lagrangian in Eq. (3) is the result of a two-loop effect, including the effective dipole interactions that originate from one loop [5]. The same conclusions hold 
for the massive dark photon since the effective $Z \gamma \bar{\gamma}$ can be induced by its own dipole-type interactions, as in Eq. (7).

Analogously, the $C P$-odd Lagrangian induced by the electric-dipole moment is instead

$$
\mathcal{L}_{\mathrm{eff}}^{(E)}=\frac{e}{\Lambda M_{Z}} C_{E} \mathcal{O}(x),
$$

where the dimension- 6 operator is

$$
\mathcal{O}(x)=Z_{\mu \nu} A^{\mu \alpha} B^{\nu}{ }_{\alpha}
$$

The expression for the coefficients $C_{E}$ in Eq. (7) is reported in the Appendix and in [5]. The operators in Eqs. (3) and (8) are $C P$ even and odd, respectively.

Concerning the decay $Z \rightarrow \gamma \bar{\gamma}$, the corresponding amplitudes in momentum space can be found in [5]. Finally, taking into account the effective Lagrangians in Eqs. (3) and (8), the total width for the unpolarized $Z$ decay is given by

$$
\Gamma(Z \rightarrow \gamma \bar{\gamma})=\frac{\alpha M_{Z}^{3}}{6 \Lambda^{2}}\left(\left|C_{M}\right|^{2}+\left|C_{E}\right|^{2}\right),
$$

where $C_{M}=\sum_{i} C_{i}$. The same results hold for the massive dark photon in the massless limit, with the scale $\Lambda$ corresponding to its dipole interactions in Eq. (7).

As discussed in [5], in the framework of a UV complete model for the dark sector, responsible for generating at one loop the dipole interactions in Eq. (7), it has been estimated that the largest allowed values for the BR could lie between $\operatorname{BR}(Z \rightarrow \gamma \bar{\gamma}) \sim 10^{-11}$ and $\operatorname{BR}(Z \rightarrow \gamma \bar{\gamma}) \sim 10^{-9}$ depending on the values of $\alpha_{D}$, the $U(1)_{D}$ coupling in the dark sector, and the $d_{M, E}^{f}$ couplings in the dipole-type interactions in Eq. (7). However, these upper limits could be relaxed if a nonperturbative dynamics is responsible for these couplings potentially pushing up the BR close to the LEP upper bound of $\operatorname{BR}(Z \rightarrow \gamma \bar{\gamma}) \simeq 10^{-6}$.

As mentioned in the Introduction, the best place to study this kind of signature is at the $e^{+} e^{-}$colliders. In particular, these BRs are in the ballpark of sensitivity of future $Z$ factories at $e^{+} e^{-}$colliders, like, for example, the FCC-ee colliders [3]. Assuming a collected number $N_{Z}=10^{13}$ of $Z$-boson events at the FCC-ee, an expected $10^{2}-10^{4}$ number of $Z \rightarrow \gamma \bar{\gamma}$ events would be possible, depending on the dark sector couplings.

\section{B. Spin 0: ALP scalar and pseudoscalar}

Here we consider a scenario for $X$ as an ALP that can mimic the $Z \rightarrow \gamma X$ signature of a massless or ultralight dark photon. We consider both the scenarios for $X$ as massive scalar $\varphi_{S}$ and pseudoscalar $\varphi_{P}$ particles and require them to behave as missing energy in the detector.

Let us assume that this process is induced by a UV physics well above the electroweak (EW) scale. In this case, an effective low energy Lagrangian approach can be used. Then we can parametrize the gauge-invariant contribution of the lowest-dimensional operators (of dimension 5) to the corresponding effective Lagrangians as

$$
\begin{aligned}
& \mathcal{L}_{\text {eff }}^{S}=\frac{1}{\Lambda_{S}} \varphi_{S} Z_{\mu \nu} F^{\mu \nu}, \\
& \mathcal{L}_{\text {eff }}^{P}=\frac{1}{\Lambda_{P}} \varphi_{P} Z_{\mu \nu} \tilde{F}^{\mu \nu},
\end{aligned}
$$

where $\Lambda_{S, P}$ are the corresponding effective scales.

Using the Lagrangians in Eq. (12), the corresponding amplitudes $M_{S}\left(M_{P}\right)$ for the $Z$ decay into scalar (pseudoscalar) plus photon channel are

$$
Z(p) \rightarrow \gamma(k) \varphi_{A}(q)
$$

with $A=S, P$ given by

$$
\begin{aligned}
& M_{S}=\frac{i}{\Lambda_{S}} \varepsilon_{Z}^{\mu}(p) \varepsilon^{\nu \star}(k) \hat{T}_{\mu \nu}^{S}(p, k), \\
& M_{P}=\frac{i}{\Lambda_{P}} \varepsilon_{Z}^{\mu}(p) \varepsilon^{\nu \star}(k) \hat{T}_{\mu \nu}^{P}(p, k),
\end{aligned}
$$

where $\hat{T}_{\mu \nu}^{S}(p, k)=2\left(\eta_{\mu \nu}(p \cdot k)-k_{\mu} p_{\nu}\right)$ and $\hat{T}_{\mu \nu}^{P}(p, k)=$ $4 \epsilon_{\mu \nu \alpha \beta} p^{\alpha} k^{\beta}$, with $\eta_{\mu \nu}$ the Minkowski metric and $\epsilon_{\mu \nu \alpha \beta}$ the complete antisymmetric tensor. Then the corresponding total decay width in the $Z$ rest frame is

$$
\hat{\Gamma}_{A} \equiv \hat{\Gamma}\left(Z \rightarrow \gamma \varphi_{A}\right)=\frac{C_{A} M_{Z}^{3}}{24 \pi \Lambda_{S}^{2}}\left(1-r_{A}\right)^{3},
$$

with $A=S, P$, where $C_{S}=1$ and $C_{P}=4$, and $r_{A}=m_{A}^{2} / M_{Z}^{2}$, with $m_{A}$ the mass of the scalar or pseudoscalar particle.

Now we consider some phenomenological implications of these results to get a feeling with the expected BRs for the $Z \rightarrow \gamma \varphi_{A}$ decays. If we assume the interactions in Eq. (12), then the ALP is a stable particle and automatically satisfies the missing-energy signature. However, we conservatively consider a more realistic scenario, which is more theoretically justified. In particular, we assume the ALP to be effectively coupled, in addition to Eq. (12), two photons with the same strength as in Eq. (12), and require that it decays (in two photons) outside the detector.

Let us focus only on the scalar case since the pseudoscalar scenario should give comparable bounds. Toward this aim, we consider in addition to Eq. (12), the existence of a new effective coupling to two photons in the Langrangian as

$$
\mathcal{L}_{\text {eff }}^{S} \supset \frac{1}{\Lambda_{S}^{\gamma \gamma}} \varphi F_{\mu \nu} F^{\mu \nu} .
$$


The reason to also consider the two photon interaction is that, from the point of view of a UV completion of the theory, one cannot avoid the presence of this interaction if the $Z \gamma \varphi_{S}$ coupling in Eq. (12) is present. Indeed, after the rotation into EW mass eigenstates, the two scales $\Lambda_{S}^{\gamma \gamma}$ and $\Lambda_{S}$ can be linearly related by coefficients proportional to the cosine and sine of the Weinberg angle $\theta_{W}[20,21]$. Then, apart from special UV models where one of the two couplings is tuned to cancel or be suppressed, these two scales are expected to be of the same order. The same conclusion does not hold for the Yukawa-like coupling of the ALP to fermions with respect to the effective interactions in Eqs. (12) and (16), where these two different kinds of interactions could be really independent from each other. ${ }^{3}$ To stick with the most simple but nontrivial scenario, we assume the ALP couplings to fermions to vanish or be strongly suppressed, thus not contributing to the total width. Then, since we are interested in the order of magnitude constraints on the effective scale $\Lambda_{S}$, we assume for simplicity $\Lambda_{S}^{\gamma \gamma} \sim \Lambda_{S}$ and set to zero all other ALP couplings to SM fields.

Under this setup, we can now analyze the constraints on the scalar or pseudoscalar mass against the corresponding effective scale $\Lambda$, which come from the requirement that the ALP does not decay inside the detector. Following the above considerations, the total width of a scalar $X$ as ALP is given by

$$
\hat{\Gamma}(S \rightarrow \gamma \gamma)=\frac{m_{S}^{3}}{16 \pi \Lambda_{S}^{2}},
$$

where $m_{S}$ is the mass of the scalar ALP.

By requiring that the ALP does not decay inside the detector, which we conservatively take to be of length $L=10 \mathrm{~m}$ for $e^{+} e^{-}$colliders, and assuming $\hat{\Gamma}(S \rightarrow \gamma \gamma)$ as the total width of ALP, we get

$$
\Lambda_{S} \gtrsim 47\left(\frac{m_{S}}{100 \mathrm{MeV}}\right)^{2} \mathrm{TeV}
$$

However, for masses below $m_{S}<100 \mathrm{MeV}$, stronger limits on the effective scale $\Lambda_{S}$ from astrophysics and low energy experiments apply that are of the order of $\Lambda_{S}>$ $10^{5}-10^{6} \mathrm{TeV}[20,21]$. These can largely exceed the bounds in Eq. (18) with stronger constraints on $\Lambda_{S}$. These lower bounds imply that $\operatorname{BR}(Z \rightarrow \varphi \gamma)<$ $10^{-13}\left(10^{-16}\right)$, corresponding to $\Lambda_{S}>10^{5}\left(10^{6}\right) \mathrm{TeV}$. As we can see, these BRs are too small to be detected, even for

\footnotetext{
${ }^{3}$ As an example, notice that the effective scales in Eqs. (12) and (16) could also be generated in the absence of Yukawa couplings of the ALP to SM fermions, induced, for instance, by new heavy messenger scalar fields (EW charged and with trilinear couplings to ALP) running in the loop.
}

the high statistics of $Z$ that could be produced at the future FCC-ee collider.

Finally, we consider the next range of $m_{S}$ massesnamely, from $100 \mathrm{MeV}$ up to the $\mathcal{O}(1 \mathrm{GeV})$-where the kinematic properties of a neutrinolike $X$ signature might still hold, assuming that the detector uncertainties do not allow one to resolve $X$ masses below $1 \mathrm{GeV}$. In this range of mass, there is still an unconstrained region of the effective scale $\Lambda_{S, P}$ from the searches at the LEP, leading to allowed values of the order of $\Lambda_{S, P}>\mathcal{O}(1 \mathrm{TeV})$ scale [20,21], which would imply a viable $\mathrm{BR}$ of the order of $\operatorname{BR}(Z \rightarrow \varphi \gamma) \sim 10^{-4}$. However, these constraints hold under the assumption that the scale $\Lambda_{S, P}$ is of the same order as the $\Lambda_{\gamma \gamma}$ one and for visible ALP decays into two photons.

On the other hand, the bound in Eq. (18) gives a stronger constraint on the effective scale $\Lambda_{S}$, which now reads $\Lambda_{S}>$ $4.7 \times 10\left(10^{3}\right) \mathrm{TeV}$ for $m_{S} \simeq 0.1(1) \mathrm{GeV}$, corresponding to a $\mathrm{BR}$ of the order of $\mathrm{BR}(Z \rightarrow \varphi \gamma) \simeq 1.8 \times 10^{-6}\left(10^{-10}\right)$. This bound is consistent with the upper limits of $10^{-6}$ on the BR from LEP negative searches of this signature [4]. As we can see, these BRs are even larger than the expected ones in $Z \rightarrow \gamma \bar{\gamma}$, and thus, potentially, larger than candidates for the signature in Eq. (1). Analogous conclusions, with BRs of same order, can be obtained for the pseudoscalar case.

In the left plot of Fig. 1, we summarize the results for the allowed regions (in color) of the number of expected events at $e^{+} e^{-}$colliders, based on the constraints in Eq. (18), as a function of the scalar mass $m_{S}$ in $\mathrm{MeV}$. For comparison, the upper bounds on the expected number of events for the massless dark photon, given by the two horizontal lines, are provided. They correspond to two representative choices for the relevant free parameters in the dark sector (see Sec. II A for additional details). For the results in Fig. 1, we have assumed the largest statistic of $N_{Z}=10^{13} \mathrm{Z}$ bosons expected to be collected at the FCC-ee in the center of mass energy $\sqrt{S}=91.2 \mathrm{GeV}$ at the $Z$ peak. As we can see, a large number of expected events are possible for an ALP particle, which would also allow one to study with sufficient precision angular distributions of the corresponding rates.

\section{Massive spin-2 particle}

For our last example, we consider the case of a massive spin-2 particle $X=G$, which is universally coupled to the total energy-momentum tensor $T_{\mu \nu}$ of SM fields. As in the case of a massive graviton, this coupling reads

$$
L_{G}=-\frac{1}{\Lambda_{G}} T^{\mu \nu} G_{\mu \nu}
$$

where $G_{\mu \nu}$ is the field associated with the spin-2 particle $G$. Since we assume $G_{\mu \nu}$ not to be related to gravitational 

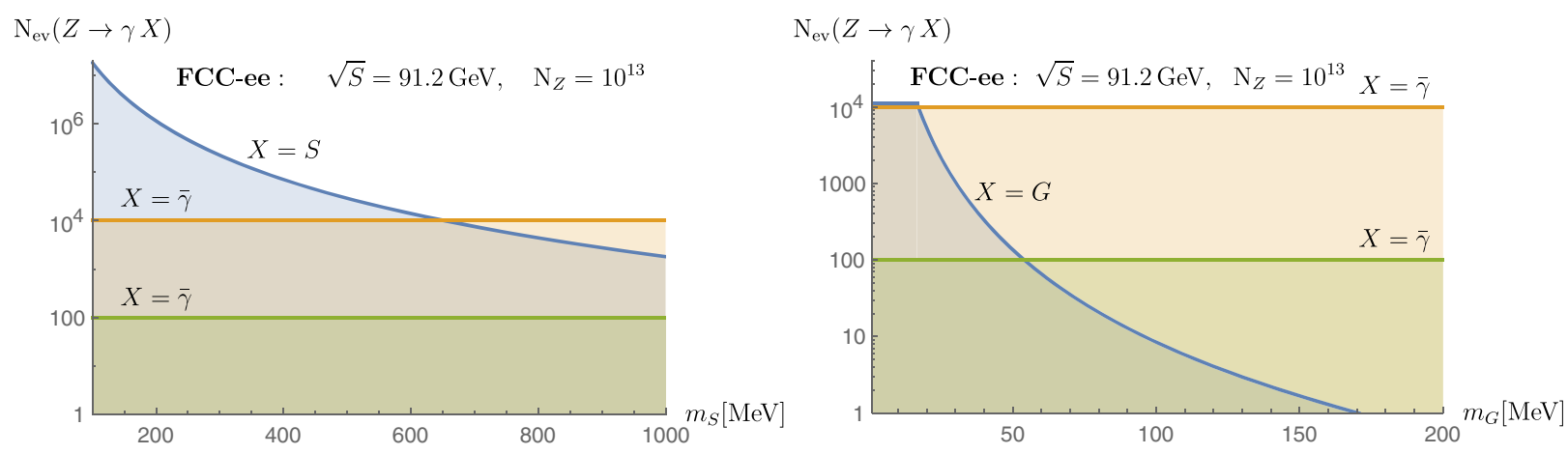

FIG. 1. Allowed regions (in color) of the number of expected events for the $Z \rightarrow \gamma X$ signal, as a function of the $m_{X}$ mass in MeV, for (left panel) $X=S$, spin-0 scalar and (right panel) $X=G$, spin-2 scalar. The two horizontal bands correspond to two representative upper bounds of the $\operatorname{BR}(Z \rightarrow \gamma \bar{\gamma})$ for the $(X=\bar{\gamma})$ massless dark photon, depending on the choice of free parameters in the dark sector (see Sec. II A for further details). These results are based on an assumption of $N_{Z}=10^{13} Z$-boson events collected at the FCC-ee in the center of mass energy $\sqrt{S}=91.2 \mathrm{GeV}$ at the $Z$ peak.

interactions, we take the effective scale $\Lambda_{G}$ as a free parameter, uncorrelated from the Planck mass, and of the order of the $\mathrm{TeV}$ scale. This scale is reduced to the usual $\Lambda_{G}^{-1}=\sqrt{8 \pi G_{N}}$ relation in the ordinary case of a massless graviton of general relativity, with $G_{N}$ the Newton constant. Since we do not make any hypothesis on the origin of the spin-2 field, we limit ourselves to the linear theory in flat space, avoiding entering into the issue of a consistent theory of massive spin-2 fields related to the nonlinear massive graviton interactions. For the purposes of this paper, the coupling in Eq. (19) is sufficient to generate a finite (thus predictive) contribution at one loop for the effective $Z G \gamma$ coupling. Indeed, owing to the fact that $G_{\mu \nu}$ is coupled to the conserved energy-momentum tensor $T^{\mu \nu}$ of matter fields, the theory is renormalizable against radiative corrections of SM matter fields only, provided the $G_{\mu \nu}$ is taken as an external field.

The free Lagrangian for the massive spin-2 particle is given by the usual term of the Fierz-Pauli Lagrangian [31] and we do not report its expression here. The corresponding Feynman rules for the $G$ interaction in Eq. (19) can be derived from previous works on massive KK graviton productions in ADD scenarios [32,33].

Now we require that the mass $m_{G}$ of the spin-2 particle is much smaller than the $Z$ mass, but larger than the $\mathrm{eV}$ scale, to avoid the strong constraints from negative searches on the Newton law deviations at short distances [29].

The effective $Z G \gamma$ coupling at low energy is generated at one loop starting from the couplings in Eq. (19), with $Z, G$, $\gamma$ the external on-shell fields, in which only virtual SM fields run inside. As mentioned above, this contribution is finite due to the conservation of $T_{\mu \nu}$ (at the zero order in $\left.1 / \Lambda_{G}\right)$. This vertex and the corresponding $Z \rightarrow \gamma G$ decay have been computed in the context of quantum gravity in large extra-dimensional scenarios [30], with $G$ the field of a generic massive spin-2 $\mathrm{KK}$ excitation of the standard graviton, and for the (massless) graviton in Einstein's theory [34].

Before entering into the discussion of the $Z \rightarrow \gamma G$ decay, we analyze the bounds on $m_{G}$ against the scale $\Lambda_{G}$, obtained by requiring that $G$ does not decay inside the detector, assumed as in Sec. II B to be of length $L=10 \mathrm{~m}$. Since we are going to discuss a light $G$ which decays into SM particles, as in the ALP case, we restrict the analysis to the range of masses

$$
\mathrm{eV} \lesssim m_{G} \lesssim 1 \mathrm{GeV}
$$

The tree-level total width of a spin-2 particle at rest, decaying into the (massless) SM fermion pair $f \bar{f}$, for the Lagrangian interaction in Eq. (19) is given by [32]

$$
\hat{\Gamma}(G \rightarrow \bar{f} f)=\frac{m_{G}^{3} N_{c}}{80 \pi \Lambda_{G}^{2}},
$$

where $N_{c}=1$ and $N_{c}=3$ for leptons and quarks, respectively, while the corresponding one for the decay into two massless gauge bosons $V$ is [32]

$$
\hat{\Gamma}(G \rightarrow V V)=\frac{N_{g} m_{G}^{3}}{40 \pi \Lambda_{G}^{2}},
$$

where $N_{V}=1$ and $N_{V}=8$ for $V=\gamma$ (photons) and $V=g$ (gluons), respectively.

Then the total width of $G$ in the visible sector, corresponding to $m_{G}=1 \mathrm{GeV}$, can be approximated as

$$
\Gamma(G \rightarrow \text { visible }) \sim 15 \hat{\Gamma}(G \rightarrow \gamma \gamma),
$$

where we have neglected all fermion masses and included channels in two photons, two gluons (assumed here to hadronize in two jets of light mesons), $e^{+} e^{-}, \mu^{+} \mu^{-}$, quark pairs $q \bar{q}$ for $q=u, d, s$. 
To simplify the analysis, we divide the range of $m_{G}$ into two regions, below and above the dimuon mass threshold $2 m_{\mu}$. In the first region, only the two photon and electron pair channel contribute to the total width. For the second region, we assume the largest value for the total width $\Gamma(G \rightarrow$ visible $)$ corresponding to $m_{G}=1 \mathrm{GeV}$, where all channels mentioned above contribute and which is quite a good approximation for our estimate. Then, by requiring that the spin-2 particle does not decay into visible states inside the detector-unlike the decay into neutrino pairs which is allowed-we get an upper bound on $m_{G}$ versus $\Lambda_{G}$ as in the ALP case-namely,

$$
\begin{array}{ll}
\Lambda_{G} \gtrsim 36\left(\frac{m_{G}}{100 \mathrm{MeV}}\right)^{2} \mathrm{TeV}, & 1 \mathrm{eV} \lesssim m_{G} \lesssim 2 m_{\mu} \\
\Lambda_{G} \gtrsim 113\left(\frac{m_{G}}{100 \mathrm{MeV}}\right)^{2} \mathrm{TeV}, & 2 m_{\mu} \lesssim m_{G} \lesssim 1 \mathrm{GeV} .
\end{array}
$$

Further theoretical constraints on this scenario should be imposed on the scale $\Lambda$ that can replace the bounds in Eq. (24) for masses below $10 \mathrm{MeV}$ with stronger constraints. In particular, to suppress potential large contributions from bremsstrahlung of $G$ in high energy experiments, which would break perturbative unitarity at the $\mathrm{TeV}$ energy colliders, we require that $\Lambda_{G}>\mathcal{O}(1 \mathrm{TeV})$ for all masses below $10 \mathrm{MeV}$. Finally, from these results we can see that for a mass range $\mathrm{eV}<m_{G} \sim 10 \mathrm{MeV}$ we have $\Lambda_{G} \gtrsim 1 \mathrm{TeV}$, while for $m_{G} \sim 50(100) \mathrm{MeV}$ we get $\Lambda_{G} \gtrsim 28(113) \mathrm{TeV}$.

Now we compute the $\operatorname{BR}(Z \rightarrow \gamma G)$ as a function of the $\Lambda_{G}$ scale. The corresponding amplitude $M_{G}$ for the process

$$
Z(p) \rightarrow \gamma(k) G(q)
$$

is induced at one loop and is given by [30]

$$
M_{G}=F_{G} \varepsilon_{Z}^{\mu}(p) \varepsilon_{G}^{\lambda \rho \star}(q) \varepsilon^{\nu \star}(k) V_{\mu \lambda \rho \nu}^{G}(k, q),
$$

where $\varepsilon_{G}^{\lambda \rho}(q)$ is the polarization tensor of the massive spin-2 field. The $F_{G}$ is a form factor which is the result of a oneloop computation. It depends only on the SM parameters. Its expression can be found in [30,34] for massive and massless $G$, respectively (with notation $F_{h}$ ). The effective vertex $V_{\mu \lambda \rho \nu}^{G}(p, q)$ is [30]

$$
\begin{aligned}
V_{\mu \lambda \rho \nu}^{G}(k, q)= & \left(k_{\lambda} q_{\nu}-(k \cdot q) \eta_{\nu \lambda}\right)\left(k_{\rho} q_{\mu}-(k \cdot q) \eta_{\mu \rho}\right) \\
& +\{\lambda \leftrightarrow \rho\} .
\end{aligned}
$$

The form factor $F_{G}$ is [30,34]

$$
F_{G} \simeq 0.41 \frac{\alpha}{\Lambda_{G} M_{Z}^{2} \pi} .
$$

After computing the square of the amplitude and summing over all polarizations, mediating by the initial ones, the unpolarized total width in the $Z$ rest frame is

$$
\hat{\Gamma}_{G}=\frac{M_{Z}^{7}}{576 \pi}\left(7+3 r_{G}\right)\left(1-r_{G}\right)^{5}\left|F_{G}\right|^{2},
$$

where $r_{G}=m_{G}^{2} / M_{Z}^{2}$, which, in the small $m_{G}$ limit, reduces to

$$
\hat{\Gamma}_{G}=\frac{7 M_{Z}^{7}}{576 \pi}\left|F_{G}\right|^{2}+\mathcal{O}\left(r_{G}\right)
$$

The result in Eq. (29) is in agreement with the corresponding one in [30]. Numerically this gives

$$
\hat{\Gamma}_{G} \simeq 2.7 \times 10^{-9}\left(\frac{1 \mathrm{TeV}}{\Lambda_{G}}\right)^{2} \mathrm{GeV}
$$

which corresponds to a branching ratio

$$
\mathrm{BR}(Z \rightarrow \gamma G)=1.1 \times 10^{-9}\left(\frac{1 \mathrm{TeV}}{\Lambda_{G}}\right)^{2} .
$$

Finally, by using the results in Eqs. (24) and (32), we find that a viable BR for the signal in Eq. (1) mediated by a long-lived spin-2 particle $G$ in the range $10^{-12} \lesssim \mathrm{BR}(Z \rightarrow$ $\gamma G) \lesssim 10^{-9}$ is possible, for a mass range between $1 \mathrm{eV}<m_{G}<50 \mathrm{MeV}$. For spin-2 masses above the $50 \mathrm{MeV}$ scale, the requirement of the missing-energy signature which is set in the upper bounds in Eq. (24) would exclude the BR above the $10^{-12}$ limit.

In the right plot of Fig. 1, the allowed regions (in color) for the number of expected events at $e^{+} e^{-}$colliders are shown as a function of the scalar mass $m_{G}$ in MeV. These bounds are based mainly on the constraints in Eq. (24) and are based on the $N_{Z}=10^{13} \mathrm{Z}$ bosons collected at the FCCee. For comparison, the two horizontal lines corresponding to the expected events in the massless dark-photon scenario, for two representative values of dark sector couplings. The flat dependence of the upper bounds of number events for the spin- 2 case corresponds to the mass-independent lower bound on the corresponding effective scale for $\Lambda_{G} \gtrsim 1 \mathrm{TeV}$ coming from negative searches of light spin-2 production at LHC, as explained above, which exceeds the lower bounds in Eq. (24) for spin-2 masses $m_{G} \lesssim 30 \mathrm{MeV}$.

\footnotetext{
${ }^{4}$ Notice that the massless limit of the width in Eq. (30) differs from the corresponding one for the pure massless graviton [34] by a overall factor $7 / 6$, which is due to the sum over polarizations of the massive graviton with respect to the massless one. This is due to the known van Dam-Veltman discontinuity in the $m_{G} \rightarrow 0$ limit [35].
} 


\section{POLARIZED PROCESSES}

Here we analyze the angular distributions for the decays $Z \rightarrow X \gamma$, summed over all polarizations of the final states, at fixed polarizations of the $Z$ boson, for the three $X$ scenarios discussed above. The reason to focus on the polarized processes is that the $Z$ boson (on shell) is always produced as polarized at colliders due to its couplings to SM fermions. We will discuss this feature in more detail in the following for the particular case of $Z$-boson production in a resonant $s$-channel at $e^{+} e^{-}$colliders.

To analyze the polarized $Z$ decays, we need to identify a special direction against which to consider its projections. In this respect, we choose a frame in which the $Z$ is boosted, and identify this direction with the one parallel to the $Z$ 3-momentum $\vec{p}_{Z}$ that we choose along the $z$-axis, in particular,

$$
p_{Z}=E_{Z}(1,0,0, \beta)
$$

where $\beta=\sqrt{1-\frac{M_{Z}^{2}}{E_{Z}^{2}}}$ is the $Z$ velocity. In this frame the differential $Z$ decay width $d \Gamma$ reads

$$
d \Gamma=\frac{|M|^{2} M_{Z}^{2}}{32 \pi E_{Z}^{3}(1-\beta z)^{2}} d z
$$

where $|M|^{2}$ is the corresponding (Lorentz-invariant) square modulus of the amplitude $z \equiv \cos \theta_{\gamma}$, with $\theta_{\gamma}$ the angle between the $Z$ and the photon 3-momenta. The distributions for the various spin cases $S_{X}=1,0,2$ in this frame are discussed below.

\section{A. Massless dark photon}

We consider first the case of a $X$ to be a massless dark photon. We anticipate here that the angular distributions of the photon for the polarized $Z$ decay induced by magneticand electric-dipole moments interactions will be the same.

We define the longitudinal $(L)$ and transverse $(T) Z$ polarizations with respect to the $Z$ momentum in Eq. (33), corresponding to the eigenstates of spin projection along the $z$ axis $J_{z}= \pm 1$ and $J_{z}=0$, respectively. Then the final result for these distributions is

$$
\begin{gathered}
\frac{1}{\hat{\Gamma}} \frac{d \Gamma^{(T)}}{d z}=\frac{3}{4}\left(\frac{M_{Z}}{E_{Z}}\right)^{5} \frac{1-z^{2}}{(1-\beta z)^{4}}, \\
\frac{1}{\hat{\Gamma}} \frac{d \Gamma^{(L)}}{d z}=\frac{3}{2}\left(\frac{M_{Z}}{E_{Z}}\right)^{3} \frac{(\beta-z)^{2}}{(1-\beta z)^{4}},
\end{gathered}
$$

where $\hat{\Gamma}$ is the total width in the $Z$ rest frame given in Eq. (10).

In Eq. (35), the distribution $d \Gamma^{(T)}$ for the transverse polarizations $J_{z}= \pm 1$ includes the average factor $(1 / 2)$ over the initial polarizations. The angular distributions corresponding to the two transverse polarization states $J_{z}= \pm 1$ are identical. As a quick check, we can see that the angular distribution in the $Z$ rest frame $(\beta=0)$ for the unpolarized process, given by

$$
\left.\frac{d \Gamma}{d z}\right|_{\beta=0}=\left(\frac{2}{3} \frac{d \Gamma^{(T)}}{d z}+\frac{1}{3} \frac{d \Gamma^{(L)}}{d z}\right)_{\beta=0}=\frac{\hat{\Gamma}}{2},
$$

is isotropic, which is in agreement with known theoretical expectations. Also, by integrating Eq. (37) at $\beta \neq 0$, the value of the total width in the moving frame $\int_{-1}^{1} d z \frac{d \Gamma}{d z}=$ $\frac{M_{Z}}{E_{Z}} \hat{\Gamma}$ is recovered.

In the $Z$ rest frame $\left(\beta \rightarrow 0, E_{Z} \rightarrow M_{Z}\right)$, where any direction is equivalent, the angle $\theta_{\gamma}$ is identified here with the angle formed between the directions of photon momentum and the $z$ axis, with the latter being the axis where the $Z$-spin projections have determined values. Then the corresponding distributions of Eqs. (35) and (36) in the $Z$ rest frame for the massless dark photon are

$$
\begin{gathered}
\frac{1}{\hat{\Gamma}} \frac{d \Gamma^{(T)}}{d z}=\frac{3}{4}\left(1-z^{2}\right), \\
\frac{1}{\hat{\Gamma}} \frac{d \Gamma^{(L)}}{d z}=\frac{3}{2} z^{2} .
\end{gathered}
$$

We will see in the next section that, due to the $Z$ couplings to the electrons, in the resonant production at $e^{+} e^{-} Z$ is mainly produced polarized at rest with transverse polarizations with respect to the beam axis.

\section{B. Scalar and pseudoscalar}

Now we repeat the same analysis as above, but in the case of $Z$ decays into a photon plus a scalar $S$ or a pseudoscalar $P$ in the massless limit. Since the polarized angular distributions for the scalar and pseudoscalar cases are the same, we will show only one of them as a representative case. Then the results for these distributions, normalized to the corresponding total width, are

$$
\begin{gathered}
\frac{1 \frac{d \Gamma_{I}^{(T)}}{\hat{\Gamma}_{I}} \frac{3}{d z}}{=}\left(\frac{M_{Z}}{E_{Z}}\right)^{3} \frac{\left(1+z^{2}\right)\left(1+\beta^{2}\right)-4 \beta z}{(1-\beta z)^{4}}, \\
\frac{1}{\hat{\Gamma}_{I}} \frac{d \Gamma_{I}^{(L)}}{d z}=\frac{3}{4}\left(\frac{M_{Z}}{E_{Z}}\right)^{5} \frac{1-z^{2}}{(1-\beta z)^{4}},
\end{gathered}
$$

with $\hat{\Gamma}_{I}$ the total width for $I=S, P$ given in Eq. (15). The distributions for the two transverse polarizations are the same. As for the spin- 1 case, one can check that, in the unpolarized case, the $Z$ the distribution in the $Z$ rest frame is independent by the angle $\theta_{\gamma}$, and that by integrating in $\theta_{\gamma}$ the total width for the unpolarized distribution in Eq. (15) is recovered. 

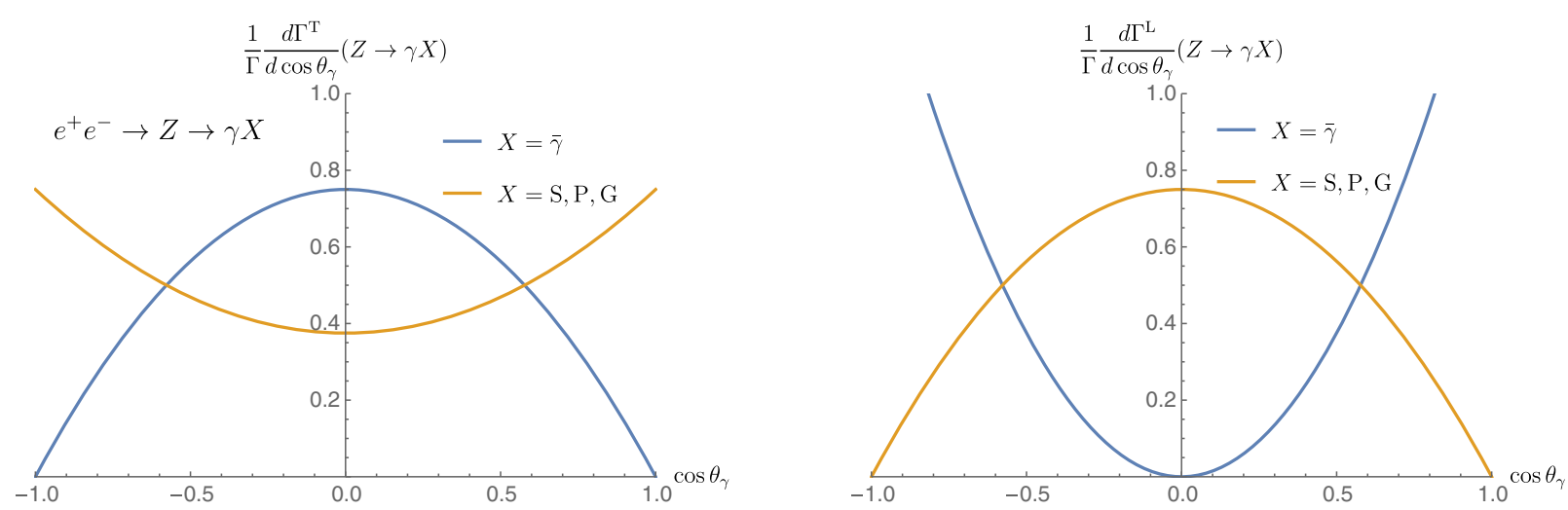

FIG. 2. Normalized distributions in $\cos \theta_{\gamma}$ for the polarized $Z \rightarrow \gamma X$ decay in the $Z$ rest frame, with $\Gamma$ the corresponding unpolarized total width, for the scenarios of $X$ as a massless dark photon $(\bar{\gamma})$, scalar/pseudoscalar $(S / P)$ and spin-2 $(G)$ particles. Here $\theta_{\gamma}$ is the angle between the directions of photon momentum and the $J_{z}$ spin axis of the $Z$ (see the text for details), The distributions of transverse $T$ and longitudinal $L$ polarizations of the $Z$, corresponding to $J_{z}= \pm 1$ and $J_{z}=0$, respectively, are shown in the left and right panels, respectively. Normalized angular distributions for a $Z$ produced at rest via $e^{+} e^{-} \rightarrow Z \rightarrow \gamma X$ are shown in the left panel, for the same $X$ scenarios, where there $\theta_{\gamma}$ is the angle between the photon momentum and the beam axis in the center of mass frame.

In the $Z$ rest frame and including also the exact $X$ mass effects, we get

$$
\begin{gathered}
\frac{1}{\hat{\Gamma}_{I}} \frac{d \Gamma_{I}^{(T)}}{d z}=\frac{3}{8}\left(1+z^{2}\right), \\
\frac{1}{\hat{\Gamma}_{I}} \frac{d \Gamma_{I}^{(L)}}{d z}=\frac{3}{4}\left(1-z^{2}\right) .
\end{gathered}
$$

Notice that these are the same distributions of the $X=S / P$ massless limit.

Remarkably, for the longitudinal and transverse polarizations, the corresponding distributions of the massless spin- 1 and spin- 0 cases are different. These distributions are shown in Fig. 2, including the spin-2 cases $X=G$.

\section{Massive spin-2 case}

Following the same analysis as above, we provide below the polarized angular distributions for the spin-2 case in the $Z \rightarrow \gamma G$ decay, in the $m_{G}$ massless limit, normalized to the corresponding total width in the $Z$ rest frame, in particular,

$$
\begin{gathered}
\frac{1}{\hat{\Gamma}_{G} \frac{d \Gamma_{G}^{(T)}}{d z}}=\frac{3}{8}\left(\frac{M_{Z}}{E_{Z}}\right)^{2} \frac{\left(1+z^{2}\right)\left(1+\beta^{2}\right)-4 \beta z}{(1-\beta z)^{4}}, \\
\frac{1}{\hat{\Gamma}_{G}} \frac{d \Gamma_{G}^{(L)}}{d z}=\frac{3}{4}\left(\frac{M_{Z}}{E_{Z}}\right)^{4} \frac{1-z^{2}}{(1-\beta z)^{4}},
\end{gathered}
$$

where the total width $\hat{\Gamma}_{G}$ is given in Eq. (30). The angular distributions for the two transverse polarizations are identical. As we can see from these results, the angular distributions of $Z$ for the spin-2 case have the same functional dependence by $\theta_{\gamma}$ of the corresponding scalar/ pseudoscalar ones at fixed polarizations; see Eqs. (40) and (41). They differ in the boosted frame only by different powers of $M_{Z} / E_{Z}$ in the overall coefficients. This equivalence holds only in the massless limit.

Below we provide the expressions for the angular distributions in the $Z$ rest frame and by retaining the exact mass dependence in $m_{G}$, in particular,

$$
\begin{gathered}
\frac{1}{\hat{\Gamma}_{G}} \frac{d \Gamma_{G}^{(T)}}{d z}=\frac{3}{8} \frac{\left(1+z^{2}\left(1-2 \delta_{G}\right)+2 \delta_{G}\right)}{1+\delta_{G}}, \\
\frac{1}{\hat{\Gamma}_{G}} \frac{d \Gamma_{G}^{(L)}}{d z}=\frac{3}{4} \frac{\left(1-z^{2}\left(1-2 \delta_{G}\right)\right)}{1+\delta_{G}},
\end{gathered}
$$

where $\delta_{G}=\frac{3}{7} r_{G}$. As we can see, spin-2 $X$ mass corrections are expected to break the equivalence between the spin-0 and spin-2 angular distributions (valid only in the $X$ massless limit) by terms of the order of $r_{G}$, which in our case are smaller than $10^{-4}$. The corresponding angular distributions for the spin- 2 distributions in the $Z$ rest frame are plotted in Fig. 2 (in the massless case).

\section{Z DECAYS AT $e^{+} e^{-}$COLLIDERS}

In this section, we will analyze the photon angular distributions coming from the $Z$-resonant process $e^{+} e^{-} \rightarrow$ $Z \rightarrow \gamma X$ at the $Z$ peak. We will show that these distributions can be easily obtained from a particular linear combination of the polarized $Z$ distributions analyzed above. This approach has the advantage to avoid the computation of the scattering cross section $e^{+} e^{-} \rightarrow Z \rightarrow \gamma X$. These results can also be applied to any final state.

In the center of mass frame of $e^{+} e^{-}$, the beam axis identifies a special direction that we choose to be our third, or $z$, axis. In this frame, we choose the initial momenta along the beam direction-namely, $p_{e^{-}}=(E, 0,0, E)$ and 
$p_{e^{+}}=(E, 0,0,-E)$, where $E=\sqrt{S} / 2$ is the center of mass energy (we neglect the electron mass). The transverse and longitudinal $Z$ polarizations for a $Z$ at rest can now be identified with respect to the beam axis.

In this frame, we define the two transverse $Z$ polarizations vectors for a $Z$ at rest as

$$
\varepsilon_{Z}^{\mu( \pm)}=\frac{1}{\sqrt{2}}(0,1, \pm i, 0)
$$

while for the longitudinal one, for a $Z$ at rest, we have

$$
\varepsilon_{Z}^{\mu(L)}=(0,0,0,1) .
$$

In the case of a frame with boosted $Z$ along the beam direction, in which $Z$ comes out with a velocity $\beta=k_{Z} / E_{Z}$, with $k_{Z}$ and $E_{Z}$ its momentum and energy, respectively, the corresponding results for the longitudinal polarization generalize to

$$
\varepsilon_{Z}^{\mu(L)}=\frac{1}{M_{Z}}\left(k_{Z}, 0,0, E_{Z}\right)
$$

Then, concerning our final state, we identify the angle $\theta_{\gamma}$ as the angle formed between the direction of the outgoing photon momentum and the initial electron momentum $\vec{p}_{e^{-}}$. In particular, for the photon 4-momentum, we have

$$
k_{\gamma}=\frac{E}{2}\left(1, \sin \theta_{\gamma} \cos \phi_{\gamma}, \sin \theta_{\gamma} \sin \phi_{\gamma}, \cos \theta_{\gamma}\right),
$$

with $\phi_{\gamma}$ the corresponding photon azimuthal angle.

Now we can extract the $\cos \theta_{\gamma}$ distributions of the final photon in $e^{+} e^{-} \rightarrow Z \rightarrow \gamma X$ by using a linear combination of the same polarized $Z$ angular distributions discussed in the previous section, provided that the $\theta$ angle appearing in the $z=\cos \theta$ distributions in Eqs. (35)-(45) is identified with the $\theta$ angle defined in Eq. (51). In this linear combination, each contribution of the $Z$ polarization $\varepsilon_{Z}^{(\lambda)}$ to the width should be multiplied by a polarization-weight coefficient $0 \leq C_{Z}^{(\lambda)} \leq 1$ (where $\sum_{\lambda= \pm, L} C_{Z}^{(\lambda)}=1$ ), corresponding to the $Z$ production in the resonance $e^{+} e^{-}$ collision.

We find these coefficients $C_{Z}^{(\lambda)}$ by performing the matching between the resonant $e^{+} e^{-} \rightarrow Z \rightarrow X_{f}$ cross section (with $X_{f}$ a generic final state) in the BreitWigner approximation against the decay width of a polarized on-shell $Z$ boson. These coefficients are universal since they depend only on the initial states, which in this case are the $e^{+} e^{-}$electron-positron pair from which the $Z$ has been created. Therefore, these results could be applied to any final state.

In general, for $e^{+} e^{-}$collisions, a generic distribution of final states $d \Gamma_{f}$ reads $d \Gamma_{f}\left(e^{+} e^{-} \rightarrow Z \rightarrow X_{f}\right)=C_{Z}^{+} d \Gamma_{f}^{+}+C_{Z}^{-} d \Gamma_{f}^{-}+C_{Z}^{L} d \Gamma_{f}^{L}$,

where $d \Gamma_{f}^{ \pm}\left(d \Gamma_{f}^{L}\right)$ stands for the corresponding transverse (longitudinal) polarized distribution of the $Z \rightarrow X_{f}$ decay and $C_{Z}^{ \pm, L}$ the corresponding polarization weights. For a $Z$ boson at rest, we have

$C_{Z}^{ \pm}=\frac{1}{2}\left(1 \mp \frac{2 g_{V}^{e} g_{A}^{e}}{\left(g_{V}^{e}\right)^{2}+\left(g_{A}^{e}\right)^{2}}\right), \quad C_{Z}^{L}=\mathcal{O}\left(m_{e} / M_{Z}\right)$,

with $m_{e}$ the electron mass. As we can see from the above results in Eq. (52), the contribution of the longitudinal polarization $\varepsilon_{Z}^{L}$ is strongly suppressed and vanishing in the limit of the vanishing electron mass. This means that the $Z$ boson produced in resonance at $e^{+} e^{-}$comes out mainly transverse polarized with respect to the beam direction. This is a well-known result that can be easily understood in terms of chirality arguments and angular momentum conservation.

The relation in Eq. (52) can be applied to all kinds of distributions of final states. In particular, it reproduces the well-known result of angular distributions of fermion pair production $e^{+} e^{-} \rightarrow Z \rightarrow f \bar{f}$ in the $Z$-resonant region at the peak, including the contribution to the forward-backward (FB) asymmetry.

In general, for a boosted frame in which the resonant $Z$ is produced with speed $\beta$ along the beam direction, the polarization coefficients $C^{ \pm}$read

$$
C^{ \pm}=\frac{1}{2}\left(1 \mp \frac{2 g_{V}^{e} g_{A}^{e}}{\left(g_{V}^{e}\right)^{2}+\left(g_{A}^{e}\right)^{2}} \frac{(1-\beta)^{2}}{1-\beta^{2}}\right) .
$$

These results could be also generalized to a resonant $Z$ production at hadron colliders via quark-antiquark annihilation, provided in Eq. (54). $g_{V, A}^{e}$ are replaced with the corresponding $g_{V, A}^{u}$ and $g_{V, A}^{d}$ couplings to up and down quarks, respectively.

The term proportional to the (干) coefficient in Eqs. (53) and (54) is responsible for the parity violating contributions. We find that, in all of the spin cases analyzed here for the $Z \rightarrow \gamma X$ process, the two angular distributions $\frac{d \Gamma^{+}}{d z}=$ $\frac{d \Gamma^{-}}{d z}$ are the same for all processes. This means that the $C^{ \pm}$ polarization coefficients enter into the combination of $C^{+}+C^{-}=1$ for a $Z \rightarrow \gamma X$ decay produced in resonance at the $e^{+} e^{-}$colliders. This is due to the fact that the $Z$ bosonic effective vertices discussed above do not introduce any parity violating contributions when $Z$ is produced from an unpolarized $e^{+} e^{-}$collider.

In conclusion, the photon angular $\left(\theta_{\gamma}\right)$ distributions coming from the resonant $Z$ boson produced in $e^{+} e^{-}$ are simply given by the $d \Gamma_{f}^{T} / d z$ expressions reported in 
Eqs. (35)-(45) and are shown in the left plot of Fig. 2 for the various $X$ scenarios.

From these results, we could see that a massless darkphoton signature is indeed characterized by a central photon, produced at large angles $\theta$ with respect to the beam, while it vanishes in the FB directions $(\theta=0, \pi)$. On the other hand, for the spin- 0 and spin- 2 cases, the photon will be mainly emitted in the FB directions. This is also in agreement with results on photon angular distributions in the KK graviton emission in the massless limit [30]. This behavior can be easily understood by angular momentum conservation. Owing to the conservation of chirality in the $Z$ couplings to initial $e^{+} e^{-}$ states, the total angular momentum $J_{Z}$ along the beam axis could be $J_{Z}= \pm 1$. On the other hand, at $\theta=0, \pi$, where orbital angular momentum vanishes, the two final photon states can have $J_{Z}=2,0$ but not $J_{Z}=0$. This forces the angular distribution rate to vanish at $\theta=0, \pi$, as shown in the left plot of Fig. 2. This conclusion does not hold for the $Z$ decay into a spin- 0 or spin-2 particle accompanied by a photon, for which the total $J_{Z}=1$ is possible at $\theta=0$, leading to a nonvanishing distribution rate in the $\mathrm{FB}$ directions.

These results suggest that, from the study of the photon angular distributions of the $Z \rightarrow \gamma X$ decay at an unpolarized $e^{+} e^{-}$collider, it would be possible to disentangle the (massless) $J^{P}=1^{-}$nature of the $X$ particle from the other $J^{P}=0^{-}, 2^{-}$hypothesis. In particular, following the results of [36], based on the search for dark-photon signal $Z \rightarrow \gamma \bar{\gamma}$ at hadronic and $e^{+} e^{-}$future colliders, the lower bound $N$ for the expected and observed numbers of signal events needed to exclude the $J^{P}=0^{-}$test hypothesis under the $J^{P}=1^{-}$assumption are, respectively, $N=6$ and $N=17$ at the $95 \%$ C.L. Combining these results with the expected number of allowed events in Fig. 1 for the massive ALP particles with these characteristics, we can conclude that there should be a sufficient number of viable events to disentangle the spin-1 versus spin- 0 hypotheses at the future linear FCC-ee.

On the other hand, it would not be possible to distinguish the $J^{P}=0^{-}$from the $J^{P}=2^{-}$signals, even by using the facility of polarized beams in the linear $e^{+} e^{-}$colliders. In general, the latter can offer great advantages in enhancing the sensitivity to new physics signal events against the SM background, as, for instance, in the search for scalar lepton partners in supersymmetry inspired models [37]. In our case, in a polarized $e^{+} e^{-}$collider one can, in principle, select the polarization weights $C^{( \pm)}$of the transverse polarizations $\varepsilon_{Z}^{( \pm)}$of the $Z$ boson in the resonant $Z$-boson production at $e^{+} e^{-}$, offering an extra tool in addition to the unpolarized circular $e^{+} e^{-}$colliders where these coefficients are fixed.
However, in the $Z \rightarrow \gamma X$ decay, because of the kind of interactions involved, the angular distributions of the $X$ spin- 0 and spin- 2 masses are identical (in the $X$ massless limit) for the two $Z$ transverse polarizations (with respect to the beam axis) $\varepsilon_{Z}^{(+)}$and $\varepsilon_{Z}^{(-)}$. Then the only way to disentangle the $X$ spin- 0 mass against the spin 2 is by using the sensitivity in the angular distribution to the $X$ mass $\left(m_{X}\right)$ effects [see Eqs. (42) and (46)], but this task can also be achieved by an unpolarized $e^{+} e^{-}$collider.

Concerning the sensitivity of the angular distributions of the spin-0 versus the spin- 2 mass, we can see that this is quite hopeless if the mass of an invisible $X$ of a spin-0 or spin- 2 mass is restricted to be below the $1 \mathrm{GeV}$ scale. On the other hand, for larger $X$ masses, the requirement to behave as an invisible particle in the detector sets quite strong constraints on the associated scale for masses larger than $1 \mathrm{GeV}$ (see the BR allowed regions in Fig. 2), thus resulting in too few (viable) events needed to analyze the angular distributions.

\section{CONCLUSIONS}

We analyzed the decays of the $Z$ boson into $Z \rightarrow \gamma X$, with $X$ a long-lived light dark boson, assumed to behave as missing energy in the detector. We discussed three potential scenarios for $X$ based on their spin origin: a massless or ultralight dark photon for the spin-1 particle, an ALP in both the scalar and pseudoscalar cases for the spin-0 particle, and a light spin- 2 particle. For the spin-0 and spin-2 scenarios, the masses are assumed to be in the range of $[100 \mathrm{MeV}-1 \mathrm{GeV}]$ for the ALP, and [1 eV$1 \mathrm{GeV}]$ for the spin-2 particle. Moreover, we required the ALP and spin-2 particles not to decay inside the detector. We showed that for these scenarios the largest BRs could be in the observable range of $10^{-12} \lesssim \mathrm{BR}(Z \rightarrow \gamma X) \lesssim$ $10^{-6}$, depending on the spin and allowed values of the corresponding effective scales. All these BRs are in the ballpark of sensitivity range of future $Z$ factories at $e^{+} e^{-}$colliders, like, for instance, the FCCee facility, with its projected production of $10^{13} \mathrm{Z}$ bosons [3].

These scenarios have in common the same signature, characterized by a monochromatic photon plus an almost neutrinolike missing energy. In case this signature should be discovered, a spin test to discriminate about the spin-1 dark-photon origin against the spin- $0 / 2$ ones was proposed. Owing to the fact that the $Z$ boson is always polarized when it is resonantly produced at $e^{+} e^{-}$colliders, we showed that the spin-1 nature of $X$ could be disentangled from the spin- 0 and spin- 2 particles by analyzing the angular distribution of the monochromatic photon. The massless dark-photon signature is indeed characterized by a photon mainly produced centrally and at large angles with respect to the $e^{+} e^{-}$beam axis. On the other hand, for the spin- 0 and spin- 2 cases (which 
have the same angular distributions) the monochromatic photon is mainly expected along the forward/backward directions.

In conclusion, due to the clean environment of the FCCee facility, together with its expectations on the high statistics of the $Z$ bosons collected, the rare $Z \rightarrow \gamma X$ decay could be a golden place to search for a light $X$ dark boson at the FCC-ee, also offering the possibility of untangling its spin origin.

\section{ACKNOWLEDGMENTS}

We thank M. Fabbrichesi, L. Marzola, B. Mele, and A. Urbano for the useful discussions. E. G. is affiliated with the Institute for Fundamental Physics of the Universe (IFPU), Trieste, Italy. E. G. thanks the Department of Theoretical Physics of CERN for its kind hospitality during the preparation of this work.

\section{APPENDIX: $Z \bar{\gamma} \bar{\gamma}$ EFFECTIVE VERTEX}

We provide here the expression of the $C_{1-3}$ and $C_{E}$ coefficients appearing in Eqs. (3) and (8) for the effective $Z \gamma \bar{\gamma}$ interactions as a function of the $d_{M, E}^{f}$ coefficient in Eq. (7). By matching the on-shell amplitude for the $Z \rightarrow \gamma \bar{\gamma}$ process-as obtained by using the effective Lagrangian in Eqs. (3) and (8) -with the corresponding one obtained by the one-loop computation with the insertion of the dipole operators in Eq. (7), we obtain [5]

$$
\begin{aligned}
& C_{1}=-\sum_{f} \frac{d_{M}^{f} X_{f}}{4 \pi^{2}}\left(5+2 B_{f}+2 C_{f}\left(m_{f}^{2}+M_{Z}^{2}\right)\right), \\
& C_{2}=-3 \sum_{f} \frac{d_{M}^{f} X_{f}}{4 \pi^{2}}\left(2+B_{f}\right), \\
& C_{3}=2 \sum_{f} \frac{d_{M}^{f} X_{f}}{4 \pi^{2}}\left(4+2 B_{f}+C_{f} M_{Z}^{2}\right)
\end{aligned}
$$

and

$$
C_{E}=\sum_{f} \frac{d_{E}^{f} X_{f}}{4 \pi^{2}}\left(3+B_{f}+2 m_{f}^{2} C_{f}\right),
$$

where $X_{f} \equiv \frac{m_{f}}{M_{Z}} N_{c}^{f} g_{A}^{f} Q_{f} e_{D}$, with $m_{f}$ the mass, $g_{A}^{f}$ the $Z$ axial coupling, $Q_{f}$ the EM charge of SM fermions $f$ in units of $e$, and $N_{c}=1$ (3) for leptons (quarks). The sum over the index $f$ runs over all EM charged SM fermions. The $B_{f}$ and $C_{f}$ terms are defined as

$$
\begin{aligned}
& B_{f} \equiv \operatorname{Disc}\left[B_{0}\left(M_{Z}^{2}, m_{f}, m_{f}\right)\right], \\
& C_{f} \equiv C_{0}\left(0,0, M_{Z}^{2}, m_{f}, m_{f}, m_{f}\right),
\end{aligned}
$$

where $B_{0}$ and $C_{0}$ are the scalar two- and three-point Passarino-Veltman functions, respectively (see [38] for their explicit expressions), and $\operatorname{Disc}\left[B_{0}\right]$ stands for the discontinuity of the $B_{0}$ function. These terms are both finite functions which can be evaluated numerically, for example, by using PACKAGE-X [39].
[1] ATLAS and CMS Collaborations, CERN Yellow Report, Reports No. CERN-LPCC-2019-01, No. CMS-FTR-19001, and No. ATL-PHYS-PUB-2019-006, 2019.

[2] R. Essig et al., arXiv:1311.0029; M. Raggi and V. Kozhuharov, Riv. Nuovo Cimento Soc. Ital. Fis. 38, 449 (2015); J. Alexander et al., arXiv:1608.08632; F. Curciarello, EPJ Web Conf. 118, 01008 (2016); A. Denig, EPJ Web Conf. 130, 01005 (2016).

[3] A. Abada et al. (FCC Collaboration), Eur. Phys. J. Special Topics 228, 261 (2019); M. Bicer et al. (TLEP Design Study Working Group), J. High Energy Phys. 01 (2014) 164; D. d'Enterria, arXiv:1602.05043.

[4] M. Acciarri et al. (L3 Collaboration), Phys. Lett. B 412, 201 (1997); O. Adriani et al. (L3 Collaboration), Phys. Lett. B 297, 469 (1992); P. Abreu et al. (DELPHI Collaboration), Z. Phys. C 74, 577 (1997); R. Akers et al. (OPAL Collaboration), Z. Phys. C 65, 47 (1995).

[5] M. Fabbrichesi, E. Gabrielli, and B. Mele, Phys. Rev. Lett. 120, 171803 (2018).

[6] J. Alexander et al., arXiv:1608.08632.

[7] M. Fabbrichesi, E. Gabrielli, and G. Lanfranchi, arXiv: 2005.01515.
[8] B. Holdom, Phys. Lett. 166B, 196 (1986); F. del Aguila, M. Masip, and M. Perez-Victoria, Nucl. Phys. B456, 531 (1995).

[9] B. A. Dobrescu, Phys. Rev. Lett. 94, 151802 (2005).

[10] E. Gabrielli, B. Mele, M. Raidal, and E. Venturini, Phys. Rev. D 94, 115013 (2016).

[11] E. Gabrielli, M. Heikinheimo, B. Mele, and M. Raidal, Phys. Rev. D 90, 055032 (2014); S. Biswas, E. Gabrielli, M. Heikinheimo, and B. Mele, J. High Energy Phys. 06 (2015) 102; Phys. Rev. D 93, 093011 (2016); 96, 055012 (2017).

[12] M. Fabbrichesi, E. Gabrielli, and B. Mele, Phys. Rev. Lett. 119, 031801 (2017).

[13] L. D. Landau, Dokl. Akad. Nauk Ser. Fiz. 60, 207 (1948); C. N. Yang, Phys. Rev. 77, 242 (1950).

[14] J. A. Dror, R. Lasenby, and M. Pospelov, Phys. Rev. Lett. 119, 141803 (2017); Phys. Rev. D 96, 075036 (2017).

[15] R. D. Peccei and H. R. Quinn, Phys. Rev. Lett. 38, 1440 (1977).

[16] E. Witten, Phys. Lett. 149B, 351 (1984).

[17] P. Svrcek and E. Witten, J. High Energy Phys. 06 (2006) 051. 
[18] A. Arvanitaki, S. Dimopoulos, S. Dubovsky, N. Kaloper, and J. March-Russell, Phys. Rev. D 81, 123530 (2010).

[19] B. S. Acharya, K. Bobkov, and P. Kumar, J. High Energy Phys. 11 (2010) 105.

[20] M. Bauer, M. Heiles, M. Neubert, and A. Thamm, Eur. Phys. J. C 79, 74 (2019).

[21] M. Bauer, M. Neubert, and A. Thamm, J. High Energy Phys. 12 (2017) 044.

[22] M. J. Dolan, T. Ferber, C. Hearty, F. Kahlhoefer, and K. Schmidt-Hoberg, J. High Energy Phys. 12 (2017) 094; I. Brivio, M. B. Gavela, L. Merlo, K. Mimasu, J. M. No, R. del Rey, and V. Sanz, Eur. Phys. J. C 77, 572 (2017); K. Mimasu and V. Sanz, J. High Energy Phys. 06 (2015) 173; J. Jaeckel and M. Spannowsky, Phys. Lett. B 753 (2016) 482; C. Baldenegro, S. Fichet, G. von Gersdorff, and C. Royon, J. High Energy Phys. 06 (2018) 131.

[23] J. Kim and U. Lee, Phys. Lett. B 233, 496 (1989).

[24] N. Arkani-Hamed, S. Dimopoulos, and G. Dvali, Phys. Lett. B 429, 263 (1998); I. Antoniadis, N. Arkani-Hamed, S. Dimopoulos, and G. Dvali, Phys. Lett. B 436, 257 (1998).

[25] L. Randall and R. Sundrum, Phys. Rev. Lett. 83, 3370 (1999); 83, 4690 (1999).
[26] A. Schmidt-May and M. von Strauss, J. Phys. A 49, 183001 (2016).

[27] K. Hinterbichler, Rev. Mod. Phys. 84, 671 (2012).

[28] C. de Rham, Living Rev. Relativity 17, 7 (2014).

[29] J. Murata and S. Tanaka, Classical Quantum Gravity 32, 033001 (2015).

[30] B. C. Allanach, J. P. Skittrall, and K. Sridhar, J. High Energy Phys. 11 (2007) 089; B. C. Allanach and J. P. Skittrall, Eur. Phys. J. C 55, 107 (2008).

[31] M. Fierz and W. Pauli, Proc. R. Soc. A 173, 211 (1939).

[32] T. Han, J. D. Lykken, and R. J. Zhang, Phys. Rev. D 59, 105006 (1999).

[33] G. F. Giudice, R. Rattazzi, and J. D. Wells, Nucl. Phys. B544, 3 (1999).

[34] J. F. Nieves and P. B. Pal, Phys. Rev. D 72, 093006 (2005).

[35] H. van Dam and M. Veltman, Nucl. Phys. B22, 397 (1970).

[36] M. Cobal, C. De Dominicis, M. Fabbrichesi, E. Gabrielli, J. Magro, B. Mele, and G. Panizzo, arXiv:2006.15945.

[37] S. Baum, P. Sandick, and P. Stengel, arXiv:2004.02834.

[38] G. Passarino and M. J. G. Veltman, Nucl. Phys. B160, 151 (1979).

[39] H. H. Patel, Comput. Phys. Commun. 197, 276 (2015); 218, 66 (2017). 